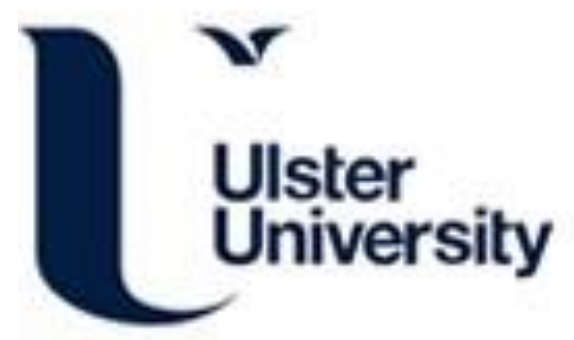

\title{
Predicting Technology Adoption in People with Dementia; Initial Results from the TAUT Project
}

Cleland, I., Nugent, C. D., McClean, S. I., Hartin, P. J., Sanders, C., Donnelly, M., Zhang, S., Scotney, B., Smith, K., Noron, M. C., \& Tschanz, J. C. (2014). Predicting Technology Adoption in People with Dementia; Initial Results from the TAUT Project. In Unknown Host Publication (Vol. 8868, pp. 266-274). Springer. https://doi.org/10.1007/978-3-319-13105-4_39

Link to publication record in Ulster University Research Portal

\section{Published in:}

Unknown Host Publication

Publication Status:

Published (in print/issue): 02/12/2014

DOI:

10.1007/978-3-319-13105-4_39

\section{Document Version}

Author Accepted version

\section{General rights}

Copyright for the publications made accessible via Ulster University's Research Portal is retained by the author(s) and / or other copyright owners and it is a condition of accessing these publications that users recognise and abide by the legal requirements associated with these rights.

\section{Take down policy}

The Research Portal is Ulster University's institutional repository that provides access to Ulster's research outputs. Every effort has been made to ensure that content in the Research Portal does not infringe any person's rights, or applicable UK laws. If you discover content in the Research Portal that you believe breaches copyright or violates any law, please contact pure-support@ulster.ac.uk. 


\title{
Predicting technology adoption in people with dementia; initial results from the TAUT project
}

\author{
Ian Cleland ${ }^{1}$, Chris Nugent ${ }^{1}$, Sally McClean ${ }^{2}$, Phillip Hartin ${ }^{1}$, Chelsea Sanders ${ }^{3}$, \\ Mark Donnelly ${ }^{1}$, Shuai Zhang ${ }^{1}$, Bryan Scotney ${ }^{2}$, Ken Smith ${ }^{4}$, \\ Maria Norton ${ }^{3}$, JoAnn Tschanz 3 . \\ ${ }^{1}$ Computer Science Research Institute and School of Computing and Mathematics, \\ University of Ulster, Newtownabbey, Co. Antrim, Northern Ireland, BT37 0QB \\ \{i.cleland, cd.nugent, mp.donnelly, s.zhang\}@ulster.ac.uk \\ $\{$ hartin-p1\}@email.ulster.ac.uk \\ ${ }^{2}$ Computer Science Research Institute and School of Computing and Information \\ Engineering, University of Ulster, Coleraine, Northern Ireland, BT52 1SA \\ \{si.mcclean, bw.scotney\}@ulster.ac.uk \\ ${ }^{3}$ Department of Phycology, Utah State University, Logan, UT 84322-4440, USA \\ \{joann.tschanz, chelsea.saunders, maria.norton, \}@usu.edu \\ ${ }^{4}$ Population Sciences, Huntsman Cancer Institute, University of Utah, Salt Lake City, UT \\ 84112, USA. \{ken.smith\}@fcs.utah.edu
}

\begin{abstract}
The acceptance of technology is a crucial factor in successfully deploying technology solutions in healthcare. Our previous research has highlighted the potential of modelling user adoption from a range of environmental, social and physical parameters. This current work aims to build on the notion of predicting technology adoption through a study investigating the usage of a reminding application deployed through a mobile phone. The TAUT project is currently recruiting participants from the Cache County Study on Memory in Aging (CCSMA) and will monitor participants over a period of 12 months. Information relating to participants' compliance with usage of the reminding application, details of cognitive assessments from the CCSMA and medical and genealogical related details from the Utah Population Database (UPDB) will be used as inputs to the development of a new adoption model. Initial results show, that with an unscreened dataset, it is possible to predict refusers and adopters with an F-measure of 0.79 .
\end{abstract}

Keywords: Technology adoption, Assistive technology, dementia, Reminding Technology.

\section{Introduction}

People with mild dementia generally exhibit impairments of memory, reasoning and thought. As a result, they require varying levels of support to complete everyday activities and to maintain a level of independence. Yet for many, a live in carer is neither practical nor affordable. Around one-third of people with dementia currently live alone without this caring presence [1]. Furthermore, the cost of providing such 
care is often unsustainable. Assistive technologies may provide an opportunity to alleviate the burden faced by Persons with dementia (PwD) and their carers, however, even with such a technology based solution a one size fits all solution remains elusive. Not everyone will be capable or willing to use the technology. Consequently, there is merit in considering a user's characteristics and specific needs when determining whether or not to recommend a form of assistive technology. Efforts to date have largely focused on the issues surrounding the technology and its perceived utility [2] whilst largely overlooking the challenges associated with people with dementia and their carers.

In this current work we aim to build upon our previous research to investigate the usage and adoption of a reminding application deployed through a mobile phone. This paper provides details of the methodology and initial results from the Technology Adoption and Usage Tool (TAUT) project which aims to model adoption and usage of assistive reminding technology for people with dementia. In section 2, a review of relevant research is provided prior to discussing the development and implementation of a smartphone app in section 3. The study protocol and initial results from the midterm analysis will then be presented in sections 4 and 5 respectively. This will include details of the profile of users who preferred not to engage with the evaluation and those who are currently using the application.

\section{Background}

The acceptance of technology is a crucial factor in successfully deploying technology solutions in healthcare and cannot be taken for granted [3]. A number of attempts been made to develop models aimed at predicting technology adoption [4, 5, $6,7]$. Originally, these models focused on the concept of perceived usefulness and ease of use [6]. Nevertheless, with increasingly diverse user backgrounds, a variety of technical solutions and use context, additional aspects may be of relevance in understanding the reasons for adopting a technology or not [7]. A common approach is to separate factors that impact upon technology adoption into external environmental factors, such as social structures, the use environment and infrastructure in addition to internal personal factors such as perceived utility, expectations and self-esteem [8]. These types of models have, however, been criticized in terms of their theoretical assumptions and practical effectiveness [2].

It is clear that there is growing academic research and societal interest in understanding factors that determine acceptance of assistive technologies for older people [9, 10, 11]. Specifically, there is demand to gain deeper insights into technology adoption through additional research. This is evidenced by the evaluations of the Whole Systems Demonstrator, which aims to build upon its existing qualitative evaluation in order to identify predictors of early removal of telehealth [12].

Our previous research in the area of technology adoption models aimed to characterize individuals with dementia and identify features that may be relevant to the adoption of assistive technology [13]. Features were collected through an iterative design process, involving evaluations with 40 participants with dementia. Features included age, gender, Mini mental state exam (MMSE) score, profession, technology 
experience and environmental conditions such as access to broadband, mobile reception and living arrangement. Based on these features, an optimal predictive model was developed. Overall, the model trained using $\mathrm{kNN}$ classification algorithm on data collected from 7 features performed the best over the four evaluation criteria of model evaluation. The model was found to maximise the opportunity of using assistive technology to allow people to stay in their home for longer and can minimize the risk of negatively impacting of mood and quality of life of the PwD and minimizing the financial risk associated with investing in assistive technology for those who do not adopt. It was noted, however, that the prediction models may have been limited by the small amount of data used for training. Given the positive results from our previous work, the current project aims to increase the amount of data available to train and test the models through engagement with a larger cohort of individuals over a longer period of time. Furthermore, through collaborations with the University of Utah and Utah State University it is possible to evaluate the use of more types of features, which we can use to develop the models. Data from the Cache County Study on Memory in Aging (CCSMA) and the Utah Population Database (UPDB) will provide further information for each participant relating to genealogical, medical, vital signs, environmental factors and demographic records.

\section{Reminder application}

The TAUT reminder application benefits from 10 years of experience in the design, implementation and evaluation of assistive cognitive prosthetics. This system has been designed by a multidisciplinary team through an iterative design process and have been previously evaluated on a small scale with a representative cohort [14]. The current version of the app, described in [15], has been developed for the android platform and is designed to provide the user with an interface to schedule and acknowledged reminders for a range of daily activities including, medication, meals, appointments and bathing. The reminders can be set by the PwD, or by a caregiver or family member and are delivered at the time specified and presented as a popup dialog box on screen accompanied by a picture indicating the type of ADL, a textual description and a melodic tone. The user has a time window of 60 seconds in which to acknowledge the reminder, after which, the popup closes, the tone stops playing and the reminder is logged as 'missed'. If acknowledged within the 60 seconds the reminder is logged as being 'acknowledged' and the popup closes. To provide additional functionality, the ability to record audio messages has also been included.

In addition to providing reminders, the TAUT application records details of the user's interactions. The app records information such as when the reminders are scheduled, when reminders are acknowledged, the type of reminder and how many reminders the person has missed. These details are then used to assess how well the user is adopting or engaging with the application in addition to providing insight into how the application is used; i.e. which activities the user requires the greatest assistance with, the most common times to receive reminders and in what form they prefer the reminder (text or voice). This data will facilitate the assessment of how users have been using the app and to what extent. Research by Hartin et al. [17] is 
investigating the context around missed reminders with the aim of providing an insight into why reminders were missed and the possibility of improving acknowledgement rates through context aware scheduling and delivery of reminders.

\section{Methods}

In order to collect the data with which to build the adoption models the project is actively recruiting participants from the CCSMA. The CCSMA is an ongoing longitudinal, population-based study of Alzheimer's disease (AD) and other dementias, which has followed over 5,000 elderly residents of Cache County, Utah (USA) since 1995. In addition, this database has been linked to the Utah Population Database (UPDB) at the University of Utah, which contains genealogical, medical, vital signs and demographic records for each of the participants, with updates made annually and with full coverage of medical information for the past 20 years. Participants have been recruited from the CCSMA to participate within the current study. At least 125 people are being recruited to partake in the study, with at least 30 participants undertaking a 12 month evaluation of the TAUT app. Some of the 125 participants will adopt the technology; others will be categorized into 3 types of nonadopter, as described in Fig. 1. In order to profile all types of non-adopter it is important to profile the users at two stages. Non-adopter (1); those who are willing to try the technology, however, for some reason are unable to use it are, profiled along with adopters using insights gained from the evaluation process. Whereas nonadopters (2) and (3) are profiled using insights gained through questionnaires delivered when the participant refuses to partake in the evaluation during the recruitment phase.

\begin{tabular}{|c|c|c|c|}
\hline \multirow{2}{*}{\multicolumn{2}{|c|}{$\begin{array}{l}\text { Mapping of adoption with two } \\
\text { factors of capability and willingness } \\
\text { to use technology }\end{array}$}} & \multicolumn{2}{|c|}{ Capable of using the technology } \\
\hline & & \multirow{2}{*}{$\begin{array}{c}\begin{array}{c}\text { Yes } \\
\text { (can use it) }\end{array} \\
\text { Adopter }\end{array}$} & \multirow{2}{*}{$\begin{array}{c}\text { No } \\
\text { (cannot use it) } \\
\begin{array}{c}\text { Non-Adopter } \\
\text { (1) }\end{array}\end{array}$} \\
\hline Willingness & $\begin{array}{c}\text { Yes } \\
\text { (want to use it) }\end{array}$ & & \\
\hline technology & $\begin{array}{c}\text { No } \\
\text { (don't want to use it) }\end{array}$ & $\begin{array}{l}\text { Non-Adopter } \\
\text { (2) }\end{array}$ & $\begin{array}{l}\text { Non-Adopter } \\
\text { (3) }\end{array}$ \\
\hline
\end{tabular}

Fig. 1. User adoption matrix showing the various types of adopter and non-adopter.

A summary of the recruitment process to date is shown in Fig. 2. Initially 335 participants were contacted by mail. 51 of these participants refused to engage at that stage of the process (non-adopter 2) and 55 where deceased. The remaining 227 were contacted by the research team by telephone; this resulted in 98 people being unreachable, 90 refusing (non-adopter 2) and 41 people agreeing to participate. Following a telephone assessment of the 41 participants who agreed, 12 are currently enrolled, 9 have agreed to participate but are currently being screened and 18 have been successfully enrolled with two participants dropping out subsequent to beginning the evaluation. Sixteen where deemed ineligible (non-adopter 3 ) due to cognitive status or currently or planning to move out of the local area in next 18 months. 


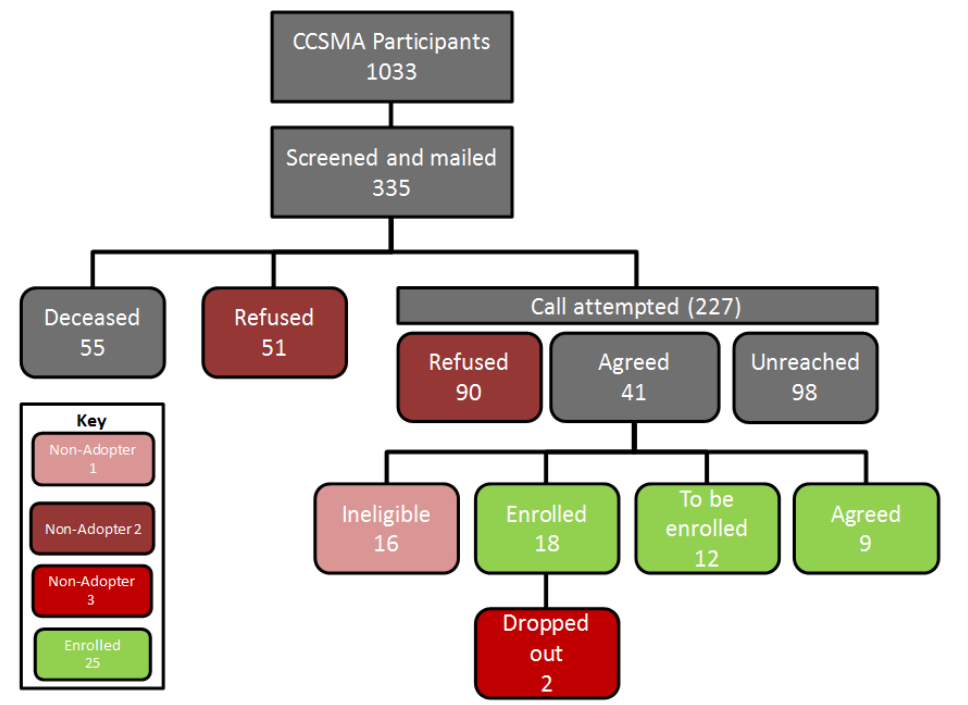

Fig.2. The recruitment process that has been undertaken and the various routes where adopters and non-adopters are obtained from.

\section{Results}

Using information gathered from participants who are currently enrolled within the study it was possible to analyze the results in a number of ways. First we considered the difference between participants who chose to be involved in the evaluation of the reminder app, following screening for eligibility, and those who refused to participate. To date, forty one people have agreed to participate in the study, although some may be ineligible due to health related factors, such as substantial memory loss. Those who agreed to partake are referred to as adopters, although they may later drop out or not engage with the technology throughout the course of the study. One hundred and forty one participants have refused to participate in the evaluation. These participants can, however, still be profiled using information from the CCSMA and the UPDB; at this stage these participants will be referred to as refusers. The following Sections will first profile each of these groups, followed by presenting the initial results from the process of modelling adoption or refusers.

\subsection{Profile of adopters and non-adopters}

Of the 335 (male=153, female=182) participants who screened as eligible for the study, the average age was 89 years. Forty one $($ male $=23$, female $=18$ ) participants agreed to participate in the study (Average age: 89 ). Two males have subsequently dropped out. One hundred and forty one $($ male $=66$, female $=75)$ participants refused to participate in the study (average age:89). There is no statistical difference in the age of the two groups $(p=0.28)$. 


\subsection{Modelling adoption and refusal}

In contrast to our previous work, we assessed the ability to classify whether or not a person was likely to agree or refuse to participate within this research study. In order to develop the most suitable model for prediction, we assessed a range of popular data mining algorithms. We also assessed the effect of feature selection on each of these algorithms, using features extracted from the CCSMA dataset only. These features focus on health and genealogy in contrast to features in previous works, which focused more on perceived utility, usefulness and experience. Data from 141 refusers and 41 adopters was used to build and test the models. Initially, 31 features (Table 1) were extracted from the CCSMA. These covered a range of areas including, age, gender, MMSE score, employment and details of a range of health conditions. Information gain (IG) was used for the purposes of feature selection. Features were ranked from highest to lowest based on IG, where a higher IG value indicates that the feature provides a better discriminative power for classification. Results showed that only 5 features had an IG greater than 0 (Last CCSMA observation $\mathrm{IG}=0.18$, APOE Genotype $\mathrm{IG}=0.156$, Any APOE4 $\mathrm{IG}=0.145$, Dementia code $\mathrm{AD}$ any $\mathrm{IG}=0.132$ and Dementia code AD pure $\mathrm{IG}=0.120$ ). APEO features describe the presence and type of the APEO/APEO4 gene. Features describing Dementia codes relate to the presence of $\mathrm{AD}$ or other forms of dementia.

Table 1. The 31 features extracted from the CCSMA database. These features where used to train the classification models.

\begin{tabular}{|c|c|c|c|c|c|}
\hline 1 & Gender & 12 & 3MS score & 23 & Stroke first observ. \\
\hline 2 & Age (Years) & 13 & 3Ms sensory adjusted (1) & 24 & Stroke Age \\
\hline 3 & Ethnicity & 14 & 3Ms sensory adjusted (2) & 25 & Hypertension self-endorsed \\
\hline 4 & APOE Genotype & 15 & 3Ms sensory adjusted (3) & 26 & Hypertension first observ. \\
\hline 5 & APEO4 copy number & 16 & Diabetes self-endorsed & 27 & Hypertension age onset \\
\hline 6 & $\begin{array}{c}\text { Any variant of } \\
\text { APOE4 }\end{array}$ & 17 & Diabetes first observ. & 28 & $\begin{array}{c}\text { High Cholesterol self- } \\
\text { endorsed }\end{array}$ \\
\hline 7 & Education level & 18 & Diabetes age onset & 29 & $\begin{array}{c}\text { High Cholesterol first } \\
\text { observ. }\end{array}$ \\
\hline 8 & $\begin{array}{c}\text { Dementia code AD } \\
\text { pure }\end{array}$ & 19 & Heart attack self-endorsed & 30 & High Cholesterol age onset \\
\hline 9 & Dementia code Any & 20 & Heart attack first observ. & 31 & Job category \\
\hline 10 & Last CCSMA observ. & 21 & Heart attack age & & \\
\hline 11 & CCSMA observ. date & 22 & Stroke self-endorsed & & \\
\hline
\end{tabular}

In order to investigate the correlation between the number of features and classifier accuracy a 10 -fold cross validation with 10 iterations was performed within Weka Experimenter (University of Waikato, Version 3.6.10). Using datasets containing subsets of 1, 2, 3, 4, 5, 10, 15, 20 and 31 ranked features. Features with the highest IG value were selected first. A range of recognized data mining algorithms for classification were selected for evaluation, namely C4.5 decision tree (DT), K-nearest neighbor $(\mathrm{kNN})$ and Naïve Bayes $(\mathrm{NB})$. To handle the data imbalance between the 
two classes, SMOTE was applied. The proportion of the data distribution was approximately $70 \%$ refusers and $30 \%$ adopters. The adopter minority class was boosted by $100 \%$. A conventional $p$-value of 0.05 was used for the threshold of significance for a paired T-test. The F-measure was used as a performance index to evaluate the performance of each of the classifiers.

Results from the analysis are presented in Fig. 3. The NB algorithm performed statistically worse than both the C4.5 and $\mathrm{kNN}$ algorithm. There was, however, no statistical difference between the C4.5 and the kNN. The DT achieved the highest Fmeasure with an average of 0.79 when using all 31 features and 85 when using 4 features, Last CCMS observation, APOE Genotype, Any variant of APOE4 and Dementia including AD any.

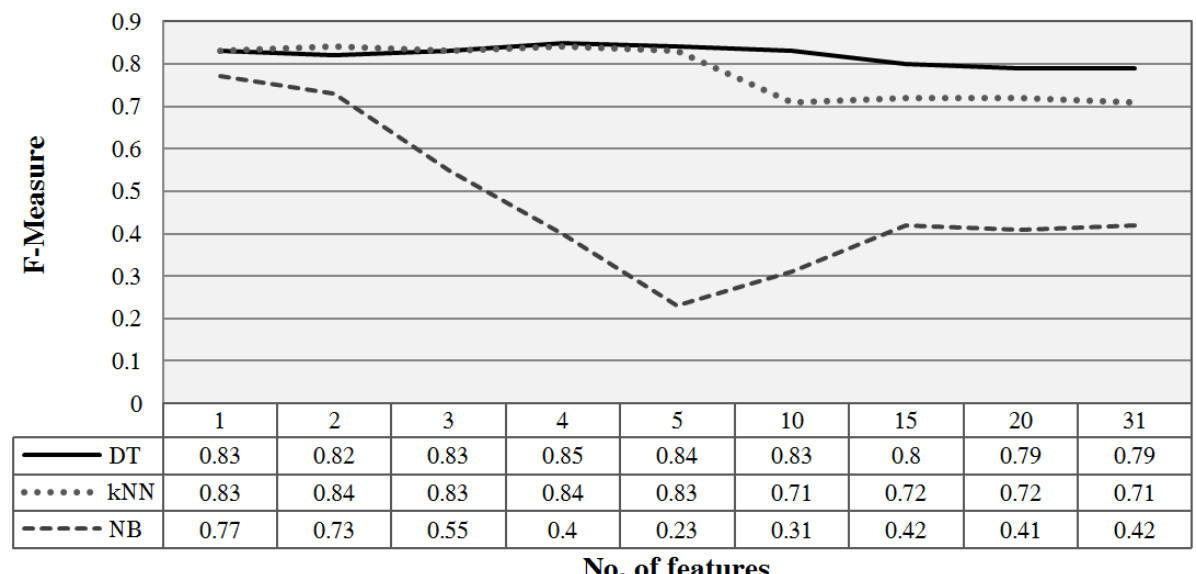

Fig 3. Graph showing the average classification F-measure for a 10 fold cross validation with 10 iterations for the $\mathrm{C} 4.5$ decision tree (DT), K-nearest neighbor $(\mathrm{kNN})$ and Naïve Bayes

(NB). Results for datasets containing 1, 2, 3, 4, 5, 10, 15, 20 and 31 features are shown.

\section{Conclusions}

Methods of predicting whether or not a user is likely to adopt an assistive technology may prove to be a powerful tool in successfully deploying technology solutions in healthcare, by reducing unnecessary costs and improving acceptance rates. This current work builds upon our previous research which has highlighted the potential of modelling user adoption from a range of environmental, social and physical parameters. Based on an initial analysis of an unscreened dataset, it was possible to predict adopters vs non-adopters with an F-measure of 0.79 using a C4.5 DT. The dataset was unbalanced, with $70 \%$ refusers to $30 \%$ adopters, this was in line with previous work [16] and the SMOTE algorithm was used to correct for this imbalance. Future work, will involve an in depth analysis of variables which can maximize the discrimination between the two classes of refusers and adopters. In addition, a closer examination will be made between the adopters and those who dropout. Furthermore, data from the CCSMA will be combined with data from the UPDB, observations from 
the evaluation and questionnaires to assess the variability in user's perceptions, to provide a further insight into the situation.

\section{Acknowledgment}

The Alzheimer's Association is acknowledged for supporting the TAUT project under the research grant ETAC-12-242841.

\section{References}

1. Kane, M., and L. Cook. "Dementia 2013: The hidden voice of loneliness." London: Alzheimer's Society (2013).

2. Chuttur, M., "Overview of the Technology Acceptance Model: Origins, Developments and Future Directions," Sprouts: Working Papers on Information Systems, vol. 9, Article 37, 2009.

3. Wilkowska, W., Gaul, S., Ziefle, M., A Small but Significant Difference - the Role of Gender on the Acceptance of Medical Assistive Technologies. In Leitner G, Hitz M, Holzinger A, editors. HCI in Work \& Learning, Life \& Leisure, 2010.

4. Day H., Jutai J., Measuring the Psychosocial Impact of Assistive Devices: the PIADS. Canadian Journal of Rehabilitation. 1996; 9(2), 159-168

5. Yen, D. C., Wu, C., Cheng, F. and Huang, Y., "Determinants of users' intention to adopt wireless technology: An empirical study by integrating TTF with TAM," Comput. Hum. Behav., vol. 26, pp. 906-915, 9, 2010.

6. Davis, F.D., Bagozzi, R.P, and Warshaw, P.R., "User Acceptance of Computer Technology: A Comparison of Two Theoretical Models," Management Science (35:8), pp. 982-1003, 1989.

7. Gaul, S., Wilkowska, W., Ziefle, M., "Accounting for User Diversity in the Acceptance of Medical Assistive Technologies." In: Szomszor, M., Kostkova, P. (Eds.): Electronic Healthcare. LNICST 69(9). Heidelberg, Dordrecht, London, New York: Springer-Verlag, pp. 175-183, 2012.

8. Scherer M.J., Jutai, J., Fuhrer, M., Demers, L. and Deruyter, F., "A framework for modelling the selection of assistive technology devices (ATDs)," Disab. \& Rehab.: Assis. Tech., vol. 2, pp. 1-8, 2007.

9. Stronge, A.J., Rogers, W.A., Fisk, A.D.J., "Human factors considerations in implementing telemedicine systems to accommodate older adults. Telemed Telecare" 13, 1-3, 2007.

10. Ziefle, M., "Age perspectives on the usefulness on e-health applications." International Conference on Health Care Systems, Ergonomics, and Patient Safety (HEPS), Straßbourg,France, 2008.

11. Arning, K., Ziefle, M., "Different Perspectives on Technology Acceptance: The Role of Technology Type and Age." In: Holzinger, A., Miesenberger, K. (eds.) USAB 2009. LNCS, vol. 5889, pp. 20-41. Springer, Heidelberg, 2009.

12. Cartwright, M., Hirani, S.P., Rixon, L., Beynon, M., H. Doll, Bower, P., et. al. "Effect of telehealth on quality of life and psychological outcomes over 12 months (Whole Systems Demonstrator telehealth questionnaire study): nested study of patient reported outcomes in a pragmatic, cluster randomized controlled trial". BMJ 2013, 346:f653, 2013.

13. Zhang, S., McClean, S. I., Nugent, C. D., et. al (2014). A predictive model for assistive technology adoption for people with dementia. IEEE journal of biomedical and health informatics, 18(1), 375.

14. O'Neill, S. A., Parente, G., Donnelly, et.al, "Assessing task compliance following mobile phone-based video reminders," in Proceedings IEEE EMBC 2011, pp5295-5298.

15. Hartin, P.J., Nugent, C.D., McClean, S.I., et. Al. A smartphone application to evaluate technology adoption and usage in persons with dementia, In Engineering in Medicine and Biology Society, EMBC, 2014 Annual International Conference of the IEEE. . pp. 5389-5392 Chicago (2014). 